\title{
Cereblon negatively regulates TLR4 signaling through the attenuation of ubiquitination of TRAF6
}

\author{
Yoon Min ${ }^{1,6}$, Sae Mi Wi ${ }^{1,6}$, Jung-Ah Kang², Taewoo Yang ${ }^{2}$, Chul-Seung Park², Sung-Gyoo Park², Sungkwon Chung ${ }^{3}$, Jae-Hyuck Shim ${ }^{4}$, \\ Eunyoung Chun ${ }^{\star, 5}$ and Ki-Young Lee ${ }^{\star, 1}$
}

Cereblon (CRBN) is a substrate receptor protein for the CRL4A E3 ubiquitin ligase complex. In this study, we report on a new regulatory role of CRBN in TLR4 signaling. CRBN overexpression leads to suppression of NF- $k B$ activation and production of proinflammatory cytokines including IL- 6 and IL- $1 \beta$ in response to TLR4 stimulation. Biochemical studies revealed interactions between CRBN and TAK1, and TRAF6 proteins. The interaction between CRBN and TAK1 did not affect the association of the TAB1 and TAB2 proteins, which have pivotal roles in the activation of TAK1, whereas the CRBN-TRAF6 interaction critically affected ubiquitination of TRAF6 and TAB2. Binding mapping results revealed that CRBN interacts with the Zinc finger domain of TRAF6, which contains the ubiquitination site of TRAF6, leading to attenuation of ubiquitination of TRAF6 and TAB2. Functional studies revealed that CRBN-knockdown THP-1 cells show enhanced NF- $k$ B activation and p65- or p50-DNA binding activities, leading to up-regulation of NF- $\kappa \mathrm{B}$-dependent gene expression and increased pro-inflammatory cytokine levels in response to TLR4 stimulation. Furthermore, $\mathrm{Crbn}^{-/}$mice exhibit decreased survival in response to LPS challenge, accompanied with marked enhancement of pro-inflammatory cytokines, such as TNF- $\alpha$ and IL-6. Taken together, our data demonstrate that CRBN negatively regulates TLR4 signaling via attenuation of TRAF6 and TAB2 ubiquitination.

Cell Death and Disease (2016) 7, e2313; doi:10.1038/cddis.2016.226; published online 28 July 2016

Cereblon (CRBN) was initially reported as a candidate gene for a mild form of autosomal recessive non-syndromic mental retardation. $^{1,2}$ Subsequently, different cellular roles of the CRBN protein have been characterized and identified. CRBN interacts with the cytoplasmic region of large-conductance calcium-activated potassium channels, regulating its surface expression. ${ }^{3}$ In the retina, CRBN interacts with voltage-gated chloride channel-2 (CIC-2), thereby influencing assembly or cellular targeting of $\mathrm{CIC}-2 .{ }^{4} \mathrm{CRBN}$ interacts with the $a 1$ subunits of AMP-activated protein kinase (AMPK) and inhibits the activation of AMPK $^{5}$ Importantly, CRBN is involved in thalidomide-induced teratogenicity. ${ }^{6}$ Thalidomide is used in the treatment of leprosy and multiple myeloma. ${ }^{7,8}$ However, its treatment is associated with limb malformations and other developmental defects. ${ }^{9,10}$ Although little is known about how these defects are caused, several reports have suggested thalidomide-induced oxidative stress and its antiangiogenic action as a possible cause of teratogenicity. ${ }^{11,12}$ Recent reports have shown that $\mathrm{CRBN}$ forms an E3 ubiquitin ligase complex and functions as a substrate receptor of the E3 ubiquitin ligase complex. ${ }^{6,13}$ Importantly, CRBN interacts with thalidomide, initiating thalidomide-induced teratogenicity by inhibiting associated ubiquitin ligase activity. ${ }^{6}$ Moreover, it has recently been reported that lenalidomide, a derivative of thalidomide, induces degradation of the lymphoid transcription factors, Ikaros and Aiolos, and casein kinase $1 a$ by $\mathrm{CRL} 4{ }^{\mathrm{CRBN}}$ E3 ubiquitin ligase, ${ }^{14}$ indicating that CRBN-binding immune modulatory drugs (IMiDs) differentially regulate CRL4 ${ }^{\mathrm{CRBN}} \mathrm{E} 3$ ubiquitin ligase activity.

So far, many different functions of E3 ubiquitin ligases have been reported. ${ }^{15-17}$ Several E3 ubiquitin ligases have a crucial role in regulating immune receptor and cellular signaling, and in modulating immune homeostasis and activation. ${ }^{18-20}$ Among them, tumor necrosis factor (TNF) receptor associated factor 6 (TRAF6), has a pivotal role in innate signaling, including signaling of toll-like receptors (TLRs). ${ }^{21,22}$ In TLRsmediated signaling, TRAF6 associates with the dimeric ubiquitin-conjugating enzyme Ubc13/Uev1A and functions

\footnotetext{
${ }^{1}$ Department of Molecular Cell Biology, Samsung Biomedical Research Institute, Sungkyunkwan University School of Medicine, 300, Cheoncheon-dong, Jangan-Gu, Suwon 440-746, Gyeonggi-Do, Republic of Korea; ${ }^{2}$ School of Life Sciences, Gwangju Institute of Science and Technology (GIST), Gwangju 500-712, Republic of Korea; ${ }^{3}$ Department of Physiology, Samsung Biomedical Research Institute, Sungkyunkwan University School of Medicine, Suwon 440-746, Republic of Korea; ${ }^{4}$ Department of Pathology and Laboratory Medicine, Weill Cornell Medical College, New York, NY 10065, USA and ${ }^{5}$ Department of Immunology and Infectious Diseases, Harvard School of Public Health, Boston, MA 02115, USA

*Corresponding author: E Chun, Department of Immunology and Infectious Diseases, Harvard School of Public Health, Boston, MA 02115, USA. Tel: +1 617 432 3250 ; Fax: +16174323259 .

or K-Y Lee, Department of Molecular Cell Biology, Samsung Biomedical Research Institute, Sungkyunkwan University School of Medicine, 300, Cheoncheon-dong, JanganGu, Suwon 440-746, Gyeonggi-Do, Republic of Korea. Tel: +82 31299 6225; Fax: +82 312996229.

E-mail: echun@hsph.harvard.edu or thylee@skku.edu

${ }^{6}$ These authors contributed equally to this work.

Abbreviations: CRBN, Cereblon; TLRs, toll-like receptors; TNF, tumor necrosis factor; TRAF6, TNF receptor associated factor 6; NF- $k B$, nuclear factor-kappa B; TAK1, transforming growth factor-beta (TGF- $\beta$ )-activated kinase 1; TAB, TAK1-binding protein; LPS, lipopolysaccharide; AMPK, AMP-activated protein kinase; CRBN ${ }^{K D}$, CRBN-knockdown; TRAF6 ${ }^{\mathrm{KD}}$, TRAF6-knockdown; Ctrl, control; Wt, wild type

Received 23.2.16; revised 28.6.16; accepted 01.7.16; Edited by T Brunner
} 
as both an adaptor and an E3 ubiquitin ligase-conjugating K63-linked ubiquitin chain attaching to itself and other proteins. ${ }^{23,24}$ TRAF6 ubiquitination involves the activation of ubiquitin-dependent kinase TAK1, along with binding to TAK1 by several different proteins, such as TAK1-binding protein (TAB)1, TAB2, TAB3, and TAB4. ${ }^{25-27}$ TAB2 is ubiquitinated by TRAF6, which facilitates assembly of a Toll/interleukin-1 (IL-1) signaling complex containing TRAF6, TAK1, and $I_{k} B$ kinase, ${ }^{27}$ leading to activation of nuclear factor-kappa $\mathrm{B}(\mathrm{NF}-\kappa \mathrm{B})$ and the production of pro-inflammatory cytokines. ${ }^{25,27}$

In this study, for the first time, we examine whether CRBN is involved in TLR4-mediated signaling through the regulation of TRAF6 ubiquitin ligase activity. CRBN interacts with the Zinc finger domain of TRAF6, which contains its ubiquitination site, and thereby critically attenuates ubiquitination of TRAF6 and TAB2, leading to inhibition of the production of proinflammatory cytokines and NF- $\kappa \mathrm{B}$-dependent gene expression. In line with our in vitro results, $\mathrm{Crbn}^{-1}$ mice exhibited increased mortality, accompanied with marked enhancements of the pro-inflammatory cytokines after challenge with lipopolysaccharide (LPS) in vivo, strongly suggesting that CRBN negatively regulates TLR4 signaling through attenuation of TRAF 6 and TAB2 ubiquitination.

\section{Results}

CRBN is negatively implicated in NF- $K B$ activation by TLR4 stimulation. To assess a possible role of CRBN in TLR4 signaling for the activation of NF- $k \mathrm{~B}$, we transfected 293/TLR4 cells with an NF- $K B$ luciferase reporter in the presence or absence of $\mathrm{HA}-\mathrm{CRBN}$. NF- $\kappa \mathrm{B}$ reporter activity was markedly increased in mock-transfected cells treated with LPS (Figure 1a, open bar versus closed bar in mock), whereas a significant decrease could be seen in CRBNtransfected cells treated with LPS (Figure 1a, closed bar in mock versus closed bar in HA-CRBN). In addition, CRBN overexpression significantly inhibited the p65-DNA binding activity induced by LPS stimulation, as compared with mock-transfected cells (Figure 1b, closed bar in mock versus closed bar in HA-CRBN). To verify the results observed in 293/TLR4 cells, we transfected THP-1 human monocytic cells with an NF- $k$ B luciferase reporter in the absence or presence of HA-CRBN. Consistently, NF- $\kappa$ B activity induced by LPS stimulation was significantly suppressed by expression of HA-CRBN, as compared to mock-transfected cells (Figure 1c, closed bar in mock versus closed bars in HA-CRBN). TLR4 stimulation induces NF- $k B$ activation, leading to the expression of pro-inflammatory cytokines. ${ }^{22,28-30}$ Accordingly, we further examined whether CRBN overexpression in THP-1 cells affected the expression of pro-inflammatory cytokines. Upon LPS stimulation in mock-transfected THP- 1 cells, IL- 6 and IL- $1 \beta$ production were greatly enhanced (Figures 1d and e, open bar versus closed bar in mock), whereas marked decreases could be detected in HA-CRBNtransfected THP-1 cells (Figures 1d and e, closed bar in mock versus closed bars in $\mathrm{HA}-\mathrm{CRBN}$ ), suggesting that CRBN overexpression negatively regulates NF- $\kappa B$ activation by TLR4 stimulation.
On the basis of the above results, we verified the functional role of CRBN in CRBN-knockdown (CRBN ${ }^{\mathrm{KD}}$ ) THP-1 cells. To generate $C R B N^{K D}$ cells, THP-1 cells were transduced with a lentivirus containing shRNA targeted to human CRBN or control shRNA sequences. Endogenous expression of CRBN protein was analyzed using a CRBN-specific antibody in both $\mathrm{CRBN}^{\mathrm{KD}}$ and control (Ctrl) THP-1 cells. CRBN expression was efficiently suppressed in CRBN ${ }^{\mathrm{KD}}$ THP-1 cells, but not in Ctrl THP-1 cells (Figure 2a). In contrast, NF- $k$ B reporter activity was significantly higher in CRBN ${ }^{\mathrm{KD}}$ THP-1 cells treated with LPS than in Ctrl THP-1 cells (Figure 2b). Furthermore, p65- or p50-DNA binding activity was also enhanced in $\mathrm{CRBN}^{\mathrm{KD}}$ THP-1 cells compared with Ctrl THP-1 cells (Figure 2c, p65 and p50). Consistently, the production of pro-inflammatory cytokines was significantly higher in CRBN ${ }^{\mathrm{KD}}$ THP-1 cells treated with LPS than in Ctrl THP-1 cells (Figure 2d, IL-6 and IL-1 $\beta$ ). To verify the function of CRBN, we transfected Ctrl and CRBN $^{\text {KD }}$ THP-1 cells with mock or Flag-tagged-CRBN (Figure $2 e$ ), and performed $\mathrm{NF}-\kappa \mathrm{B}$ reporter assay. The overexpression of Flag-CRBN in both $\mathrm{Ctrl}$ and $\mathrm{CRBN} \mathrm{KD}^{\mathrm{KD}}$ THP-1 cells led to significant inhibition of NF- $K B$ reporter activity, compared to mock-transfected cells (Figure 2f). Moreover, IL- 6 and IL-1 $\beta$ production were also significantly attenuated in both $\mathrm{Ctrl}$ and $\mathrm{CRBN}^{\mathrm{KD}} \mathrm{THP}-1$ cells overexpressed by Flag-CRBN, as compared with mocktransfected cells (Figure 2g, IL-6 and IL-1 $\beta$ ). These results strongly suggest that CRBN negatively regulates TLR4mediated signaling, thereby inhibits the activation of NF- $\kappa$ B.

CRBN interacts with TAK1, but has no effect on associations of TAB1 and TAB2 with TAK1. Next, we investigated the molecular mechanism by which CRBN negatively regulates TLR4 signaling. In TLR4 signaling, ubiquitin modification has emerged as an important mechanism that regulates cell signaling for the NF- $\kappa \mathrm{B}$ activation. ${ }^{31,32}$ TRAF6, an important E3 ubiquitin protein ligase, has a key role in the TLR4 signaling pathway. Ubiquitinated TRAF6 has been implicated in the activation of TAK1 through the ubiquitination of TAB2, eventually leading to activation of NF- $K B .^{23}$ Therefore, we assumed that CRBN could be implicated with ubiquitin-mediated activation of TLR4 signaling. Coimmunoprecipitation was used to assess the association of CRBN with various components associated with TRAF6 in TLR4 signaling, including TAK1, TAB1, and TAB2. Flag-TAK1 significantly immunoprecipitated with HA-CRBN (Figure 3a, lane 3). In contrast, HA-CRBN did not coprecipitate with FlagTAB1 or Flag-TAB2 (lane 5 in Figures $3 b$ and $c$, respectively). A consistent interaction between CRBN and TAK1 was evident (lane 4 of Figures $3 b$ and c, respectively), suggesting that CRBN interacts with TAK1, but not TAB1 or TAB2.

We further examined whether the interaction of CRBN and TAK1 could affect the association of TAB1 and TAB2 to TAK1. To do this, we determined the TAK1 interaction sites of CRBN, TAB1, and TAB2 using TAK1 truncation mutants (Figure 3d). Flag-CRBN significantly co-precipitated with Myc-TAK1 wt, Myc-TAK1 1-500, and Myc-TAK1 1-400 (Figure 3e), but not Myc-TAK1 1-200 and Myc-TAK1 1-300, suggesting that CRBN interacts with the TAK1 300-400 domain. Flag-TAB1 was significantly co-precipitated with Myc-TAK1 wt and MycTAK1-truncated mutants (Figure 3f). In contrast, Flag-TAB2 
a

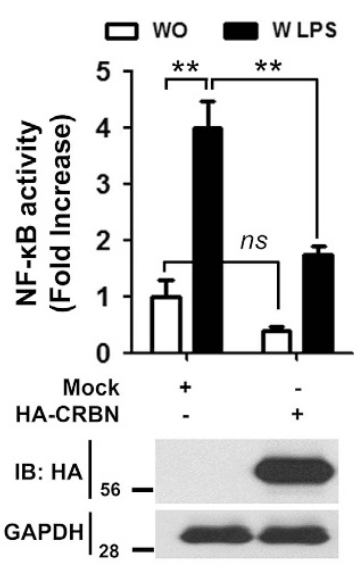

d

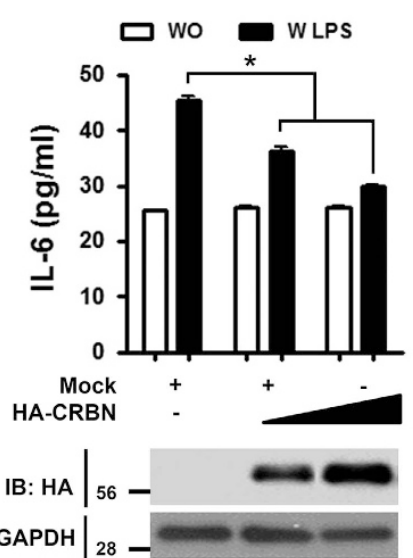

IB: GAPDH $\left.\right|_{28}=-$ b

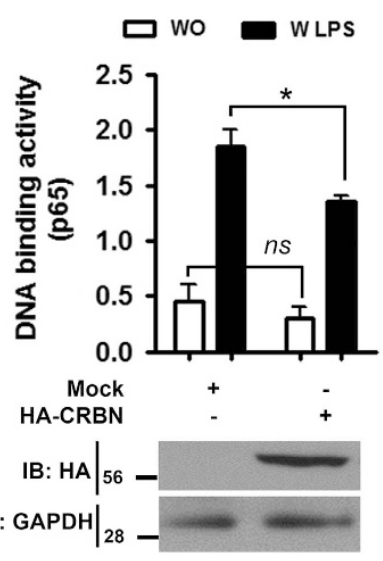

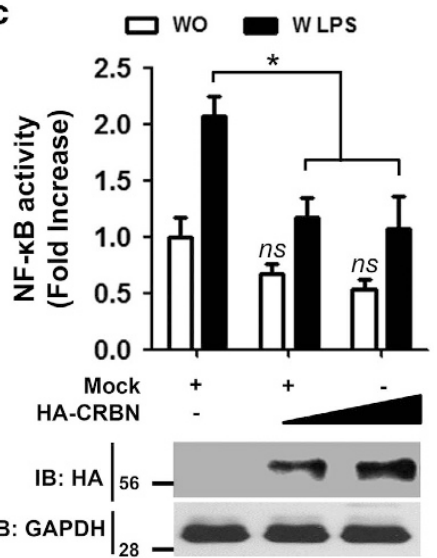

e $\square$ wo $\square$ wLS

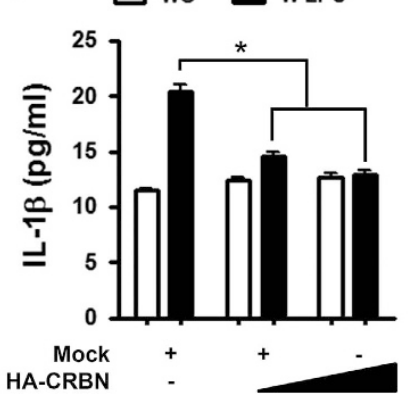

Figure 1 CRBN overexpression inhibits TLR4-induced NF- $\kappa$ B activation. (a) 293/TLR4 cells were transfected with a mock or HA-CRBN vector together with a pBIllx-luc and Renilla luciferase vector. Twenty-four hours after transfection, cells were untreated or treated with LPS $(200 \mathrm{ng} / \mathrm{ml})$ for $6 \mathrm{~h}$ and then analyzed for luciferase activity. Results are expressed as the fold induction in luciferase activity relative to that in untreated cells. All error bars represent standard deviation (S.D.) of the mean from triplicate samples. $n s$, not significant; ${ }^{* *} P<0.01$. (b) 293/TLR4 cells were transfected with a mock or HA-CRBN vector, treated with or without LPS (200 $\left.\mathrm{ng} / \mathrm{ml}\right)$ for $6 \mathrm{~h}$, and then analyzed for p65-DNA binding activity using the manufacturer's protocol. All error bars represent S.D. of the mean from triplicate samples. $n s$, not significant; ${ }^{*} P<0.05$. (c) THP-1 cells were transfected with mock or different concentrations of HA-CRBN vector together with the pBllx-luc and Renilla luciferase vectors. Twenty-four hours after transfection, cells were untreated or treated with LPS $(200 \mathrm{ng} / \mathrm{ml})$ for $6 \mathrm{~h}$ and then analyzed for luciferase activity. Results are expressed as the fold induction in luciferase activity relative to that in untreated cells. All error bars represent S.D. of the mean from triplicate samples. $n s$, not significant; ${ }^{*} P<0.05$. (d,e) THP-1 cells were transfected with mock or different concentrations of HA-CRBN vector, treated with or without LPS (200 ng/ml) for $9 \mathrm{~h}$, and production of IL-6 (d) and IL-1 $\beta$ (e) were analyzed by enzyme-linked immunosorbent assay (ELISA). All error bars represent S.D. of the mean from triplicate samples. ${ }^{*} P<0.05$

co-precipitated with only Myc-TAK1 wt (Figure 3g). These results suggest that CRBN, TAB1, and TAB2 may interact with different regions of TAK1 (Figure 3h). To directly confirm the result, Myc-TAK1 and Flag-TAB1 or Flag-TAB2 were co-transfected into HEK293T cells with different concentrations of HA-CRBN. Flag-TAB1 and Flag-TAB2 significantly co-precipitated with Myc-TAK1 in the absence or presence of different concentrations of HA-CRBN (Figures $3 i$ and j, lanes 2 and 3-6, respectively). Moreover, the interaction between TAK1 and CRBN was significantly increased in a dose-dependent manner (Figures $3 i$ and j, lanes 3-6, IB: HA). These results demonstrate that CRBN interacts with TAK1, and that the interaction does not affect the association of TAB1 and TAB2 with TAK1.

CRBN interacts with TRAF6, which attenuates TRAF6 and TAB2 ubiquitination. Having shown that CRBN has no effect on the formation of the TAK1-TAB1-TAB2 complex, we next examined whether CRBN affects the formation of the TRAF6-TAB2 complex. An IP assay revealed that Flag-TRAF6 significantly co-precipitated with HA-CRBN (Figure $4 a$, lane 3 ). To identify the interaction domain of TRAF6 in CRBN, truncated TRAF6 mutants were generated (Figure 4b; Supplementary Table 1), and then co-transfected into HEK293T cells along with HA-CRBN. HA-CRBN was coprecipitated with Flag-TRAF6 wt and Flag-TRAF6 110-522 (Figure 4c, lanes 1 and 2), but not with Flag-TRAF6 260-522 or Flag-TRAF6 349-522, suggesting that CRBN interacts with the zinc finger domain of TRAF6. K124 in TRAF6 is required for IL-1-dependent ubiquitination and IKK activation. ${ }^{24}$ As K124 is located in the zinc finger domain of TRAF6, we assessed whether the interaction of CRBN with the zinc finger domain of TRAF6 would affect the ubiquitination of TRAF6. Flag-TRAF6 was co-transfected into HEK293T cells along with Myc-CRBN in the presence or absence of HA-Ub. TRAF6 ubiquitination was quite apparent in the absence of Myc-CRBN, whereas marked attenuation was noted in the 
a
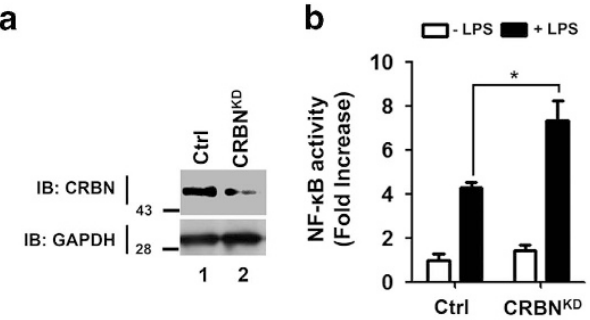

C

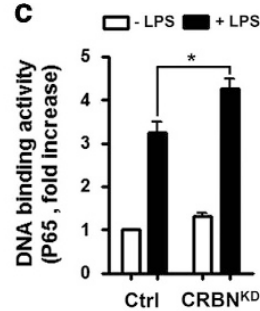

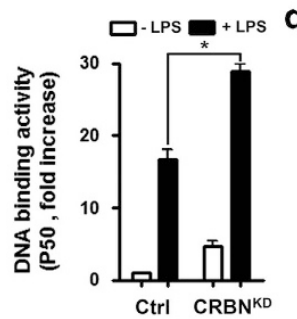
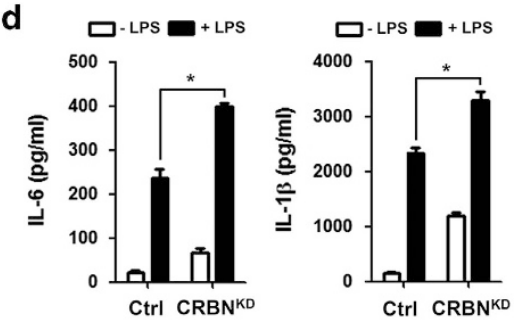

e

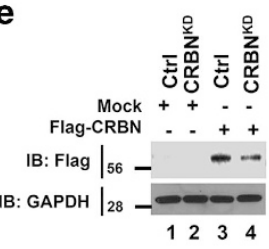

$\mathbf{f}$

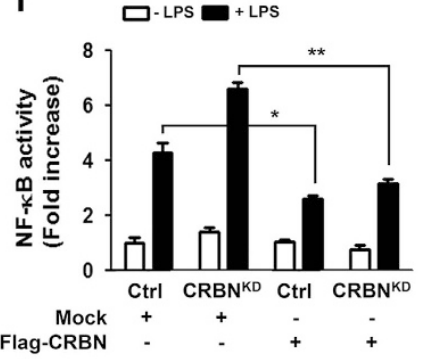

g

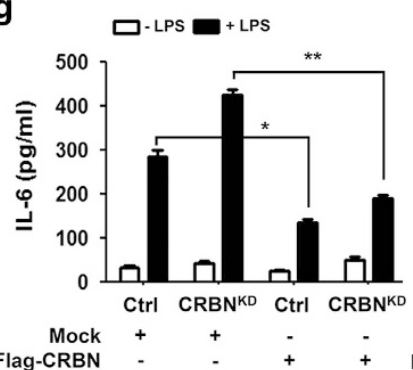

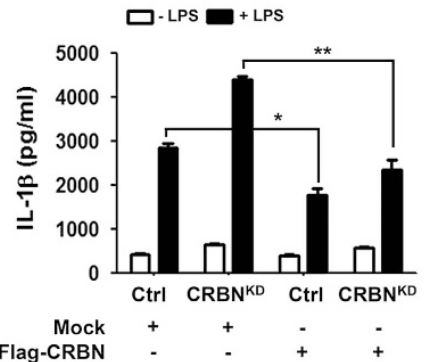

Figure 2 CRBN-knockdown THP-1 cells exhibit increased NF-kB activity and production of pro-inflammatory cytokines. (a) THP-1 cells were infected with a lentivirus containing shRNA targeted to human CRBN or control shRNA. Two weeks post-infection, endogenous expression of CRBN proteins were analyzed in CRBN ${ }^{\mathrm{KD}}$ and control (Ctrl) THP-1 cells. (b) CRBN ${ }^{\mathrm{KD}}$ and Ctrl THP-1 cells were transfected with the pBllx-luc and Renilla luciferase vectors. Twenty-four hours after transfection, cells were untreated or treated with LPS $(200 \mathrm{ng} / \mathrm{ml})$ for $6 \mathrm{~h}$ and then analyzed for luciferase activity. Results are expressed as the fold induction in luciferase activity relative to that in untreated cells. All error bars represent S.D. of the mean from triplicate samples. ${ }^{*} P<0.05$ (c) CRBN ${ }^{\mathrm{KD}}$ and Ctrl THP-1 cells were treated with or without LPS (200 ng/ml) for $6 \mathrm{~h}$ and then analyzed for p65- or p50-DNA binding activity using the manufacturer's protocol. All error bars represent S.D. of the mean from triplicate samples. ${ }^{*} P<0.05$ (d) CRBN ${ }^{K D}$ and Ctrl THP-1 cells were treated with or without LPS (200 ng/ml) for $9 \mathrm{~h}$, and production of IL- 6 and IL-1 $\beta$ were analyzed by ELISA. All error bars represent S.D. of the mean from triplicate samples. ${ }^{*} P<0.05$. (e) $\mathrm{CRBN}^{\mathrm{KD}}$ and Ctrl THP-1 cells were transfected with a mock or Flag-CRBN vector, as indicated, and then Flag-CRBN expression was examined by western blotting. (f) $\mathrm{CRBN}^{\mathrm{KD}}$ and Ctrl THP-1 cells were transfected with a mock or Flag-CRBN vector (as shown in (e)) together with pBllx-luc and Renilla luciferase vectors. Twenty-four hours after transfection, cells were untreated or treated with LPS $(200 \mathrm{ng} / \mathrm{ml})$ for $6 \mathrm{~h}$ and then analyzed for luciferase activity. The results are expressed as the fold induction in luciferase activity relative to that in untreated cells. All error bars represent S.D. of the mean from triplicate samples. ${ }^{*} P<0.05$ and ${ }^{* *} P<0.01$. (g) $C R B N^{K D}$ and Ctrl THP-1 cells were transfected with a mock or Flag-CRBN vector (as shown in (e)), treated with or without LPS (200 ng/ml) for $9 \mathrm{~h}$, and production of IL-6 and IL-1 $\beta$ were analyzed by ELISA. All error bars represent S.D. of the mean from triplicate samples. ${ }^{*} P<0.05$ and ${ }^{* *} P<0.01$

presence of Myc-CRBN (Figure 4d, lane 5 versus lane 8). To verify the result, Flag-TRAF6 and HA-Ub were co-transfected into HEK293T cells with different concentrations of MycCRBN. According to expressions of Myc-CRBN, the ubiquitination of TRAF6 gradually decreased (Figure 4e, lane 2 versus lanes 3-5), indicating that CRBN attenuates the ubiquitination of TRAF6.

On the basis of the above results, we examined whether CRBN is negatively involved in the TRAF6-induced activation of NF-KB in response to TLR4 stimulation. To do this, we generated TRAF6-knockdown (TRAF6 ${ }^{\mathrm{KD}}$ ) THP-1 cells using a lentivirus containing shRNA targeted to human TRAF6 (Figure 4f). Ctrl or TRAF6 ${ }^{\mathrm{KD}}$ THP-1 cells were transiently transfected with mock, Flag-TRAF6, and HA-CRBN, as indicted in Figure 4g. As expected, TRAF6 ${ }^{\mathrm{KD}}$ THP-1 cells displayed decreased NF- $K B$ reporter activity in response to LPS stimulation, as compared with Ctrl cells (Figure 4g, column 2 versus column 8). In addition, overexpression of TRAF6 significantly enhanced NF-kB reporter activity in both cells (Figure 4g, column 2 versus column 3 in Ctrl; column 8 versus column 9 in TRAF6 ${ }^{\mathrm{KD}}$ ). Interestingly, TRAF6induced activation of NF- $\kappa B$ was significantly attenuated by the expression of CRBN in a dose-dependent manner (Figure 4g, column 3 versus columns 4, 5, and 6 in Ctrl; column 8 versus columns 10,11 , and 12 in TRAF6 ${ }^{\mathrm{KD}}$ ). Production of the cytokine IL- 6 was also markedly enhanced in both groups of cells transfected with Flag-TRAF6 (Figure 4h, column 2 versus column 3 in Ctrl; column 6 versus column 7 in in TRAF6 ${ }^{\mathrm{KD}}$ ), whereas the effect was significantly suppressed in the cells co-transfected with Flag-TRAF6 and HA-CRBN (Figure 4h, column 3 versus column 4 in Ctrl; column 7 versus column 8 in in TRAF6 ${ }^{\mathrm{KD}}$ ), strongly suggesting that CRBN functionally regulates TRAF6 through attenuation of ubiquitination.

Ubiquitinated TRAF6 may activate TAK1 through ubiquitination of TAB2. ${ }^{23,33}$ Therefore, we examined whether the effect of CRBN on TRAF6 ubiquitination leads to the ubiquitination of TAB2. Flag-TAB2 was co-transfected into HEK293T cells along with Myc-CRBN in the presence or absence of HA-Ub. The ubiquitination of TAB2 was significantly apparent in the absence of Myc-CRBN, whereas marked attenuation was evident in the presence of Myc-CRBN (Figure 5a, lane 5 versus lane 8). To verify this result, Flag-TAB2 and HA-Ub were co-transfected into HEK293T cells with different concentrations of Myc-CRBN. The ubiquitination of TAB2 was gradually decreased in a Myc-CRBN dose-dependent manner (Figure 5b, lane 2 versus lanes 3-6). As ubiquitination of TRAF6 and TAB2 is critically important for the activation of the IKK complex, ${ }^{23,33}$ we therefore examined whether a deficiency in CRBN positively regulates the activation of IKKs in response to TLR4 stimulation. In order to do that, $\mathrm{CRBN}^{+/+}$ or $\mathrm{CRBN}^{-1}$ MEF cells were stimulated without or with LPS for different times, as indicated in Figure 5c. Interestingly, phosphorylated IKKa $\beta$ was significantly elevated in $\mathrm{CRBN}^{-/}$ MEF cells in response to LPS stimulation, and that led to the 

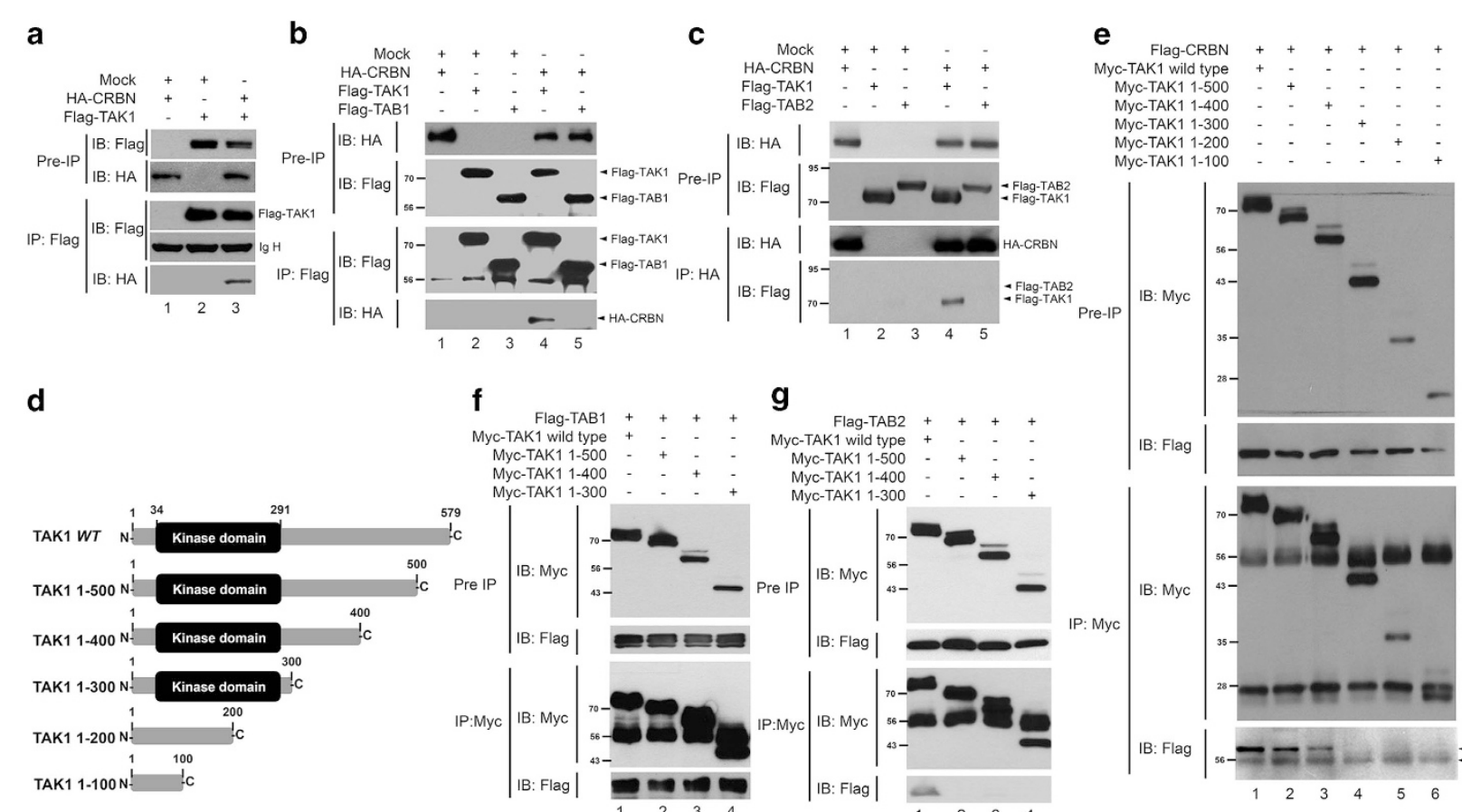

h
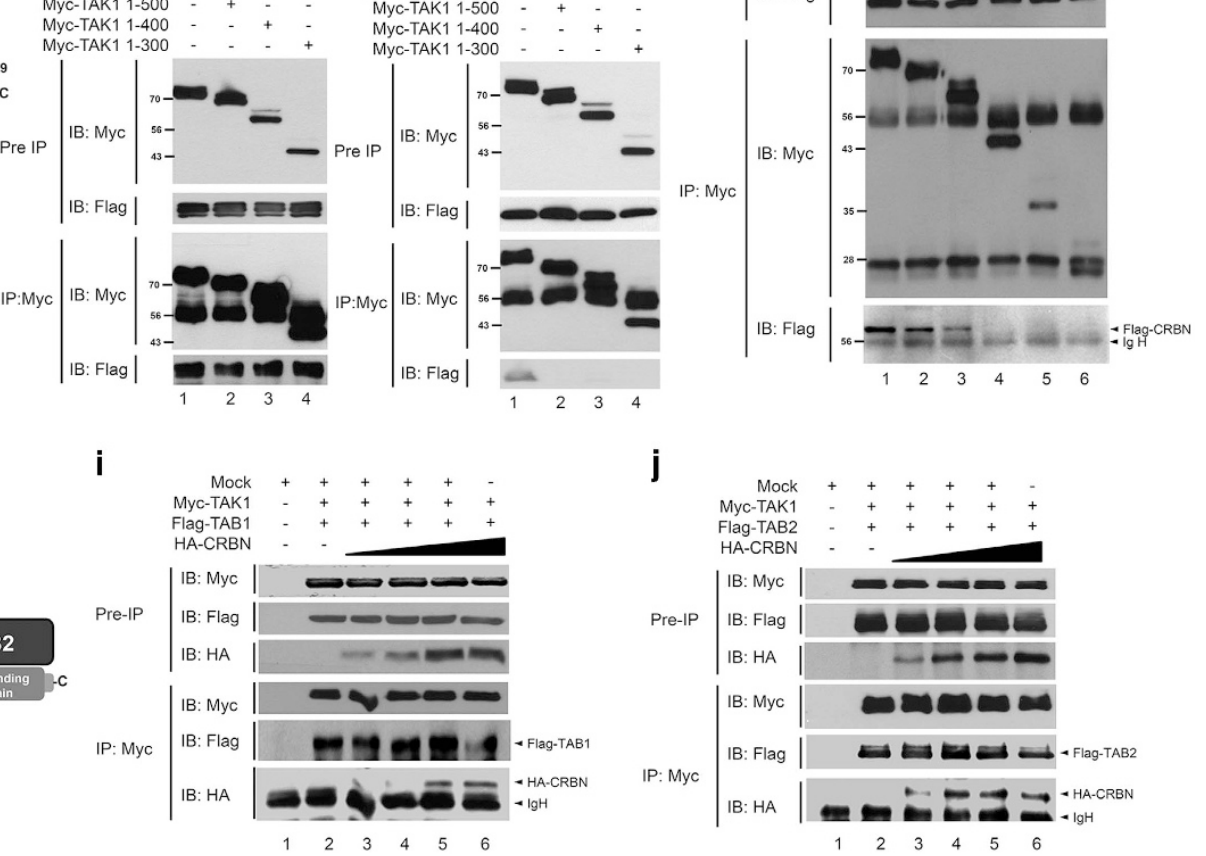

Figure 3 CRBN interacts with TAK1, but not with TAB1 or TAB2. (a) HEK293T cells were transfected with mock, HA-CRBN, or Flag-TAK1, as indicated. At $38 \mathrm{~h}$ after transfection, transfected cells were extracted, immunoprecipitated with the anti-Flag antibody, and immunoblotting was performed with the anti-Flag or anti-HA antibodies (b,c) HEK293T cells were transfected with mock, HA-CRBN, Flag-TAK1, Flag-TAB1, or Flag-TAB2, as indicated in each figure. At $38 \mathrm{~h}$ after transfection, transfected cells were extracted, immunoprecipitated with anti-Flag (b) or anti-HA (c) antibodies, and immunoblotting was performed with anti-Flag or anti-HA antibodies. (d) TAK1 wild type (wt) and TAK1-truncated mutants: TAK1 1-500, TAK1 1-400, TAK1 1-300, TAK1 1-200, and TAK1 1-100. (e) HEK293T cells were transfected with Myc-TAK1 wt or Myc-TAK1 1-500, MycTAK1 1-400, Myc-TAK1 1-300, Myc-TAK1 1-200, or Myc-TAK1 1-100, along with Flag-CRBN. At $38 \mathrm{~h}$ after transfection, transfected cells were extracted, immunoprecipitated with anti-Myc, and then immunoblotting was performed with anti-Flag or anti-Myc antibodies. (f,g) HEK293T cells were transfected with Myc-TAK1 wt or Myc-TAK1 1-500, Myc-TAK1 1-400, Myc-TAK1 1-300, Flag-TAB1 (f), or Flag-TAB2 (g), as indicated in each figure. At $38 \mathrm{~h}$ after transfection, transfected cells were extracted, immunoprecipitated with anti-Myc antibody, and then immunoblotting assay was performed with anti-Flag or anti-Myc antibodies. (h) A schematic model of the interaction between TAK1 and TAB1, TAB2, or CRBN. (i,j) HEK293T cells were transfected with Myc-TAK1 and Flag-TAB1 (i) or Myc-TAK1 and Flag-TAB2 (j) in the presence or absence of different concentrations of HA-CRBN. At $38 \mathrm{~h}$ after transfection, transfected cells were extracted, immunoprecipitated with an anti-Myc antibody, and then immunoblotting was performed with anti-Flag, anti-HA, or anti-Myc antibodies

degradation of $\mathrm{IKB}_{\mathrm{K}} \boldsymbol{a}$, which was not the case in $\mathrm{CRBN}^{+/+} \mathrm{MEF}$ cells (Figure $5 \mathrm{c}, \mathrm{CRBN}^{-/-}$versus $\mathrm{CRBN}^{+/+} \mathrm{MEF}$ cells), suggesting that the inhibition of TRAF6 and TAB2 ubiquitination by CRBN is negatively associated with IKK activation. To functionally verify the results, Ctrl or TRAF6 ${ }^{\mathrm{KD}}$ THP-1 cells were transiently transfected with mock, Flag-TRAF6 wt, FlagTAB2, and HA-CRBN constructs, as indicted in Figure $5 \mathrm{~d}$, and then cells were stimulated with or without LPS. Overexpression of TRAF6 and TAB2 significantly enhanced NF-KB reporter activity in both cell types (Figure $5 \mathrm{~d}$, column 2 versus column 4 in Ctrl; column 7 versus column 7 in TRAF6 ${ }^{\mathrm{KD}}$ ). Interestingly, the TRAF6 and TAB2-induced activation of
NF- $\kappa$ B was significantly attenuated by expression of CRBN (Figure $5 \mathrm{~d}$, column 4 versus column 5 in Ctrl; column 9 versus column 10 in TRAFG ${ }^{\mathrm{KD}}$ ). In terms of the production of IL-6, similar results were evident in both cell types (Figure 5e), indicating that the negative effect of CRBN on TRAF6 ubiquitination is linked to suppression of TAB2 ubiquitination, which leads to inhibition of NF-kB activation.

CRBN negatively regulates NF-KB-dependent gene expression and septic shock response to LPS challenge. Finally, we examined the functional role of CRBN in ex vivo and in vivo systems. Microarray analysis was done to assess 


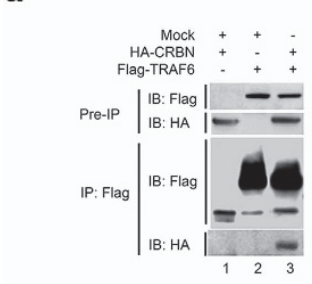

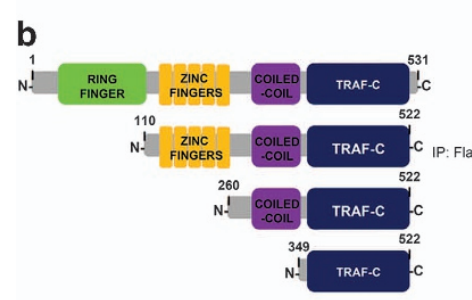

f

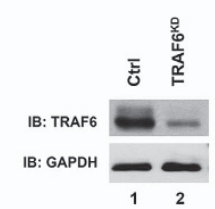

g

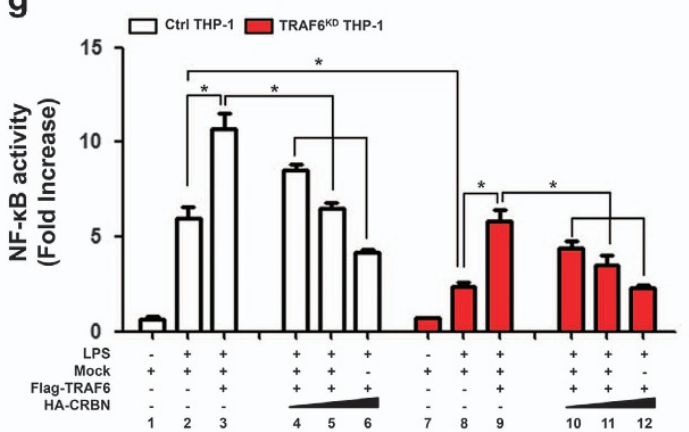

c

d

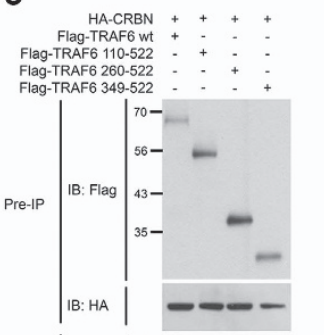

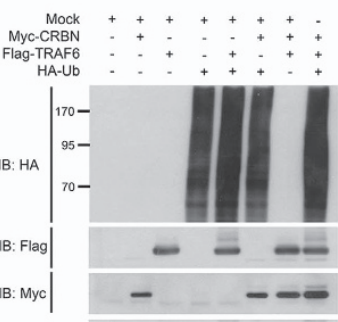

e
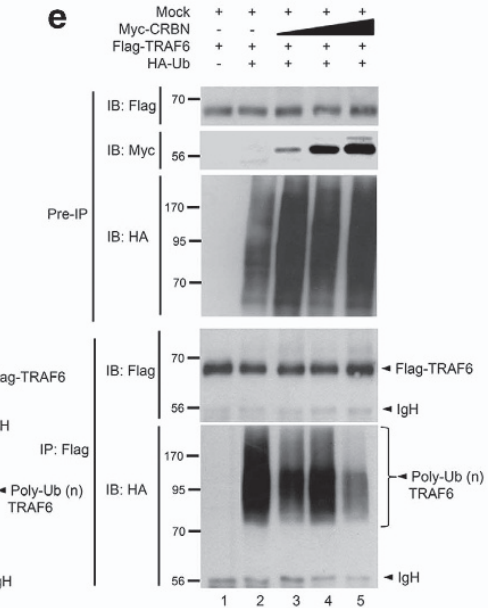

h

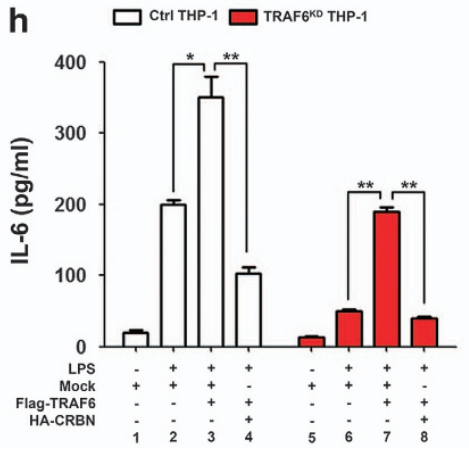

Figure 4 CRBN interacts with TRAF6 and inhibits the ubiquitination of TRAF6. (a) HEK293T cells were transfected with mock, HA-CRBN, or Flag-TRAF6, as indicated. At $38 \mathrm{~h}$ after transfection, transfected cells were extracted, immunoprecipitated with anti-Flag antibody, and then immunoblotting was performed with anti-Flag or anti-HA antibodies. (b) A schematic presentation of TRAF6 wt and its truncated mutants used in this study: TRAF6 110-522, TRAF6 260-522, and TRAF6 349-522. (c) HEK293T cells were transfected with HA-CRBN, Flag-TRAF6 wt, Flag-TRAF6 110-522, Flag-TRAF6 260-522, or Flag-TRAF6 349-522, as indicated. At $38 \mathrm{~h}$ after transfection, transfected cells were extracted, immunoprecipitated with an anti-Flag antibody, and then immunoblotting was performed with anti-Flag or anti-HA antibodies. (d) HEK293T cells were transfected with mock, Myc-CRBN, Flag-TRAF6, or HA-Ub, as indicated. At $38 \mathrm{~h}$ after transfection, transfected cells were extracted, immunoprecipitated with an anti-Flag antibody, and then immunoblotting was performed with anti-Flag, anti-Myc, or anti-HA antibodies. (e) HEK293T cells were transfected with mock, HA-Ub, and Flag-TRAF6 in the absence or presence of different concentrations of Myc-CRBN. At $38 \mathrm{~h}$ after transfection, transfected cells were extracted, immunoprecipitated with an anti-Flag antibody, and then immunoblotting was performed with anti-Flag, anti-Myc, or anti-HA antibodies. (f) THP-1 cells were infected with a lentivirus containing shRNA targeted to human TRAF6 or control shRNA sequences. Two weeks post-infection, endogenous expression of TRAF6 protein was analyzed in TRAF6 ${ }^{\mathrm{KD}}$ and control (Ctrl) THP-1 cells. (g) TRAF6 ${ }^{\mathrm{KD}}$ and Ctrl THP-1 cells were transfected with mock, Flag-TRAF6, or HA-CRBN vectors, as indicted, together with pBllx-luc and Renilla luciferase. Twenty-four hours after transfection, cells were untreated or treated with LPS (200 ng/ml) for $6 \mathrm{~h}$ and then analyzed for luciferase activity. Results are expressed as the fold induction in luciferase activity relative to that in untreated cells. All error bars represent S.D. of the mean from triplicate samples. ${ }^{*} P<0.05$. (h) TRAF6 ${ }^{K D}$ and Ctrl THP-1 cells were transfected with mock, Flag-TRAF6, or HA-CRBN vectors, as indicated, and treated with or without LPS $(200 \mathrm{ng} / \mathrm{ml})$ for $9 \mathrm{~h}$, then, production of IL-6 was analyzed by ELISA. All error bars represent S.D. of the mean from triplicate samples. ${ }^{*} P<0.05$ and ${ }^{* *} P<0.01$

whether CRBN knockdown in THP-1 cells is functionally able to regulate NF-KB-dependent genes induced by TLR4 stimulation. Following stimulation with LPS, marked changes in gene expression profiles could be detected (Supplementary Figure 1). To assess NF- $k \mathrm{~B}-d e p e n d e n t$ gene expression with LPS stimulation, NF- $\kappa$ B-dependent genes containing specific $\kappa$ B-binding DNA sequences were further sorted out. Expression levels of genes were significantly altered in Ctrl or CRBN ${ }^{\mathrm{KD}}$ THP-1 cells not treated or treated with LPS (Figure 6a). To verify the results, quantitative real-time PCR (qRT-PCR) analysis was done using specific primers targeted to the IL-1 $\beta$, IER3, BCL2, CCL5, IL-8, and IRF7 genes (Figures $6 \mathrm{~b}-\mathrm{g}$ ). These genes were significantly upregulated in LPS-treated Ctrl THP-1 cells, as compared with non-stimulated cells (Figures 6b-g, without LPS versus with LPS in Ctrl cells). Moreover, gene expression was markedly elevated in CRBN ${ }^{\mathrm{KD}}$ THP-1 cells compared with LPS-treated Ctrl THP-1 cells (Figures 6b-g, open bars versus closed bars), indicating that $\mathrm{CRBN}$ negatively regulates NF-kB-dependent gene expression induced by TLR4 stimulation.

Next, we examined whether the mortality rate in CRBNdeficient mice $\left(\mathrm{Crbn}^{-/-}\right)$is critically affected by LPS challenge, and whether the effects are associated with increases in levels of pro-inflammatory cytokines. CRBN-deficient $\left(\mathrm{Crbn}^{-/-}\right)$and control mice $\left(\mathrm{Crbn}^{+/+}\right)$were challenged with LPS $(7.5 \mathrm{mg} / \mathrm{kg}$ intraperitoneally), and then the survival rate was monitored over time. Following LPS challenge, the higher mortality rate in $\mathrm{Crbn}^{-/-}$mice was significantly greater than that of $\mathrm{Crbn}^{+/+}$ mice (Figure $7 \mathrm{a}, \mathrm{Crbn}^{-/-}$versus $\mathrm{Crbn}^{+/+}$). Half of the $\mathrm{Crbn}^{-/-}$ 
a

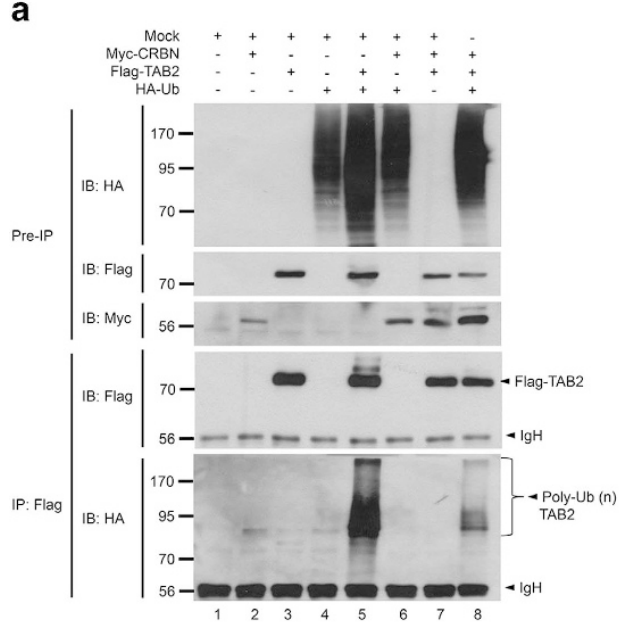

d

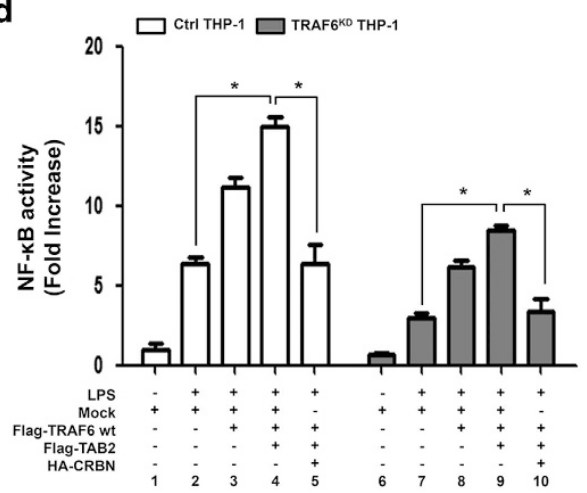

b
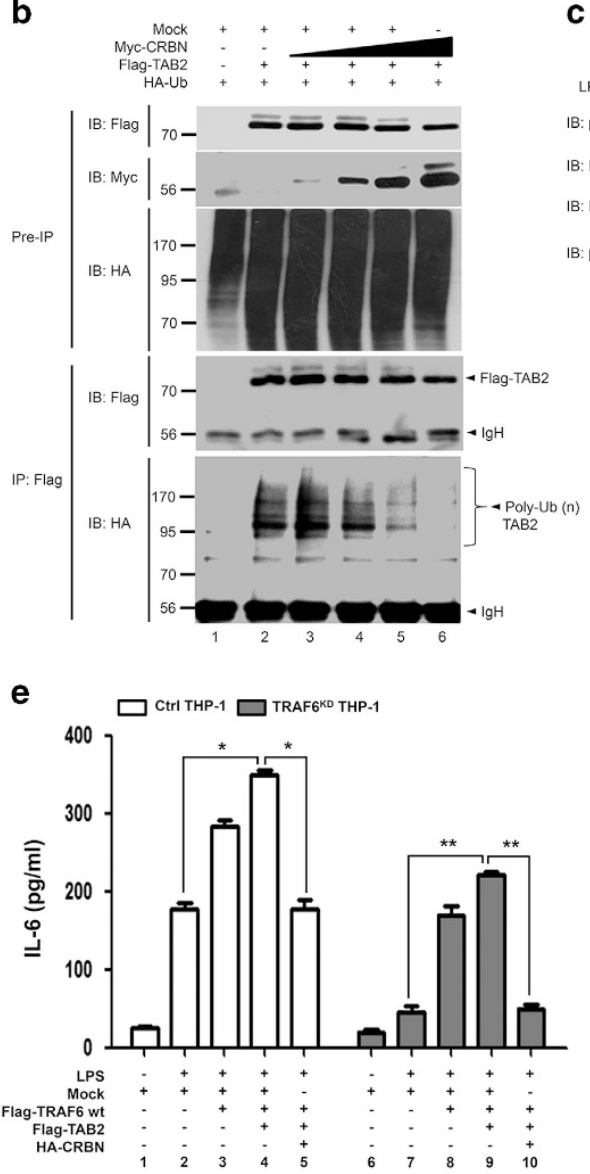
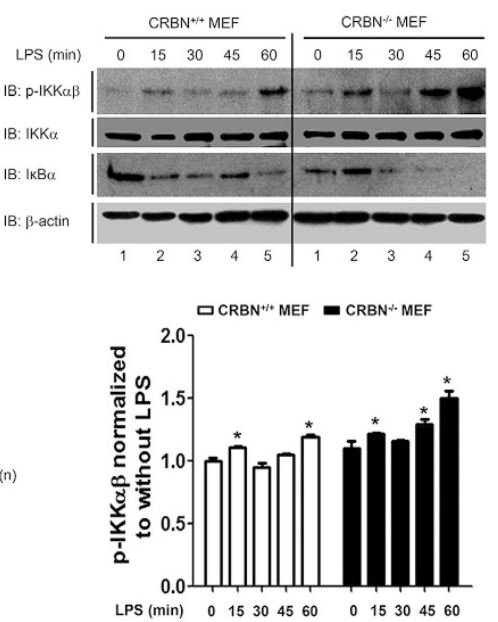

Figure 5 CRBN inhibits ubiquitination of TAB2. (a) HEK293T cells were transfected with mock, Myc-CRBN, Flag-TAB2, or HA-Ub, as indicated. At $38 \mathrm{~h}$ after transfection, transfected cells were extracted, immunoprecipitated with anti-Flag antibody, and then immunoblotting was performed with anti-Flag, anti-Myc, or anti-HA antibodies. (b) HEK293T cells were transfected with mock, HA-Ub, and Flag-TAB2 in the absence or presence of different concentrations of Myc-CRBN. At 38 h after transfection, transfected cells were extracted, immunoprecipitated with an anti-Flag antibody, and then immunoblotting was performed with anti-Flag, anti-Myc, or anti-HA antibodies. (c) $\mathrm{CRBN}^{+/+}$or $\mathrm{CRBN}^{-/}$MEF cells were stimulated without or with LPS for different periods of time, as indicated, then subjected to extraction, and western blotting was performed with the antibodies indicated on the left. The band intensity of $\mathrm{p}-\mathrm{IKK} \alpha / \beta$ was analyzed with Image $\mathrm{J}$ (bottom). All error bars represent S.D. of the mean from three independent experiments ${ }^{*} P<0.05$ versus without LPS. (d) TRAF6 ${ }^{\mathrm{KD}}$ and Ctrl THP-1 cells were transfected with mock, Flag-TRAF6 wt, Flag-TAB2, or HA-CRBN vectors, as indicted, together with pBIlxluc and Renilla luciferase. Twenty-four hours after transfection, cells were untreated or treated with LPS (200 ng/ml) for $6 \mathrm{~h}$ and then analyzed for luciferase activity. Results are expressed as the fold induction in luciferase activity relative to that in untreated cells. All error bars represent S.D. of the mean from triplicate samples. ${ }^{*} P<0.05$. (e) TRAF $6^{\mathrm{KD}}$ and Ctrl THP-1 cells were transfected with mock, Flag-TRAF6 wt, Flag-TAB2, or HA-CRBN vectors, as indicted, and treated with or without LPS (200 ng/ml) for $9 \mathrm{~h}$, and production of IL-6 was analyzed by ELISA. All error bars represent S.D. of the mean from triplicate samples. ${ }^{\star} P<0.05$ and ${ }^{* \star} P<0.01$

mice died within 4 days of LPS challenge, whereas $90 \%$ of $\mathrm{Crbn}^{+/+}$mice survived for this period of time (Figure 7a). In addition, $100 \%$ mortality of $\mathrm{Crbn}^{-1-}$ mice occurred within 6 days of LPS challenge, whereas $40 \%$ of $\mathrm{Crbn}^{+/+}$mice survived for more than 6 days (Figure 7a), indicating that $\mathrm{Crbn}^{-1-}$ mice are more susceptible to LPS challenge. To examine whether mortality is associated with an enhancement in serum levels of pro-inflammatory cytokines, TNF- $a$ and IL-6 levels were measured in response to LPS exposure. The levels of both cytokines were significantly higher in $\mathrm{Crbn}^{-1-}$ mice than in $\mathrm{Crbn}^{+/+}$mice (Figure 7b, TNF-a; Figure 7c, IL-6). These results strongly suggest that $\mathrm{Crbn}^{-1-}$ mice exhibit a higher mortality rate following LPS challenge, and the effects may be critically related to the production of pro-inflammatory cytokines, such as IL- 6 and TNF- $a$, which are regulated by $\mathrm{NF}-k \mathrm{~B}$.

\section{Discussion}

We provide evidence that CRBN negatively regulates TLR4mediated signaling. Biochemical studies to deduce the molecular mechanism revealed that CRBN interacts with TAK1 and TRAF6, but not TAB1 and TAB2. As the association of TAB1 and TAB2 proteins to TAK1 is critically implicated with TAK1 activation, ${ }^{25-27}$ we examined whether the interaction of CRBN and TAK1 affects the molecular association of TAB1 and TAB2. We found that CRBN does not interrupt the association between TAB1 and TAB2 to TAK1 (Figure 3h). Interestingly, CRBN interacted with TRAF6 through the zinc finger domain of TRAF6. Regarding the ubiquitination of TRAF6 in TLR4 signaling, the autoubiquitination site (K124) of TRAF6 is located in the zinc finger domain and is functionally critical for the association of the TAK1-TAB2 complex. ${ }^{24}$ 
a

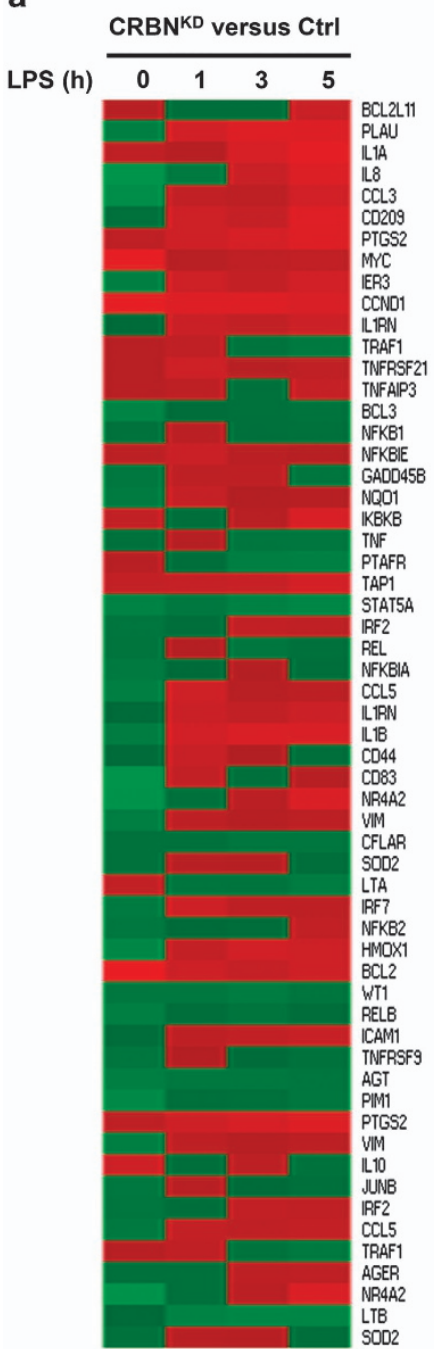

b
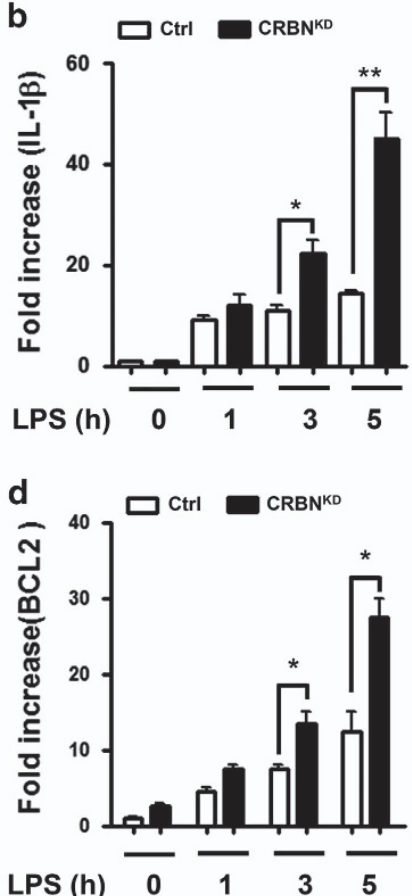

$\mathbf{f}$

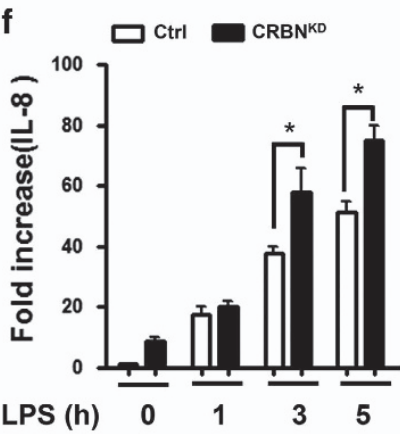

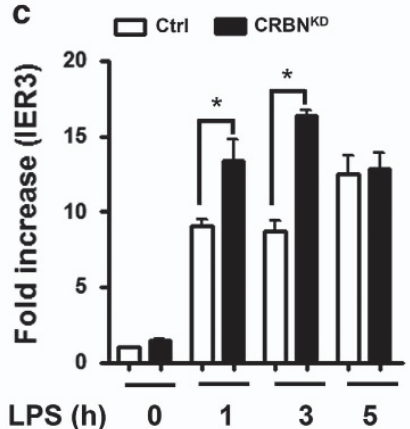
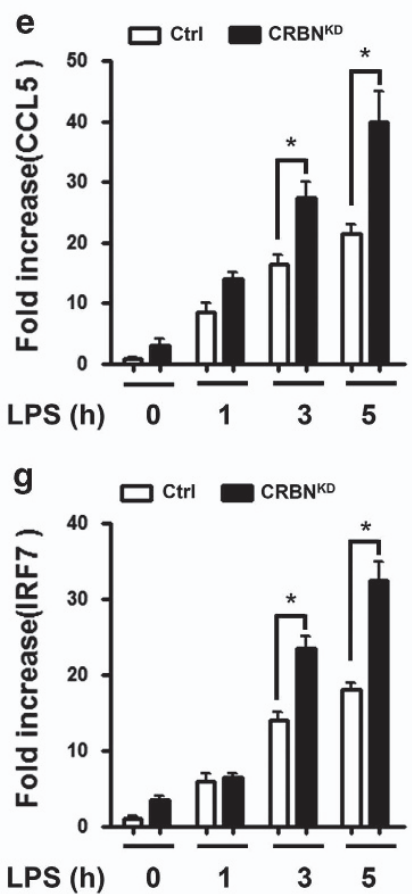

Figure 6 CRBN-knockdown THP-1 cells enhance NF- $k B$-dependent gene expression. (a) Ctrl THP-1 and CRBN ${ }^{K D}$ THP-1 cells were treated with or without LPS (200 ng/ml) for different lengths of time, as indicated. NF- $\kappa B$-dependent gene expression was compared at each time point (CRBN ${ }^{K D}$ versus Ctrl THP-1). (b-g) RNA was extracted from Ctrl and CRBN ${ }^{\mathrm{KD}}$ THP-1 cells treated with or without LPS $(200 \mathrm{ng} / \mathrm{ml})$ for different periods of time, as indicated, followed by qRT-PCR analysis with primers specific for the IL-1 $\beta(\mathbf{b})$, IER3 (c), BCL2 (d), CCL5 (e), IL-8 (f), and IRF7 (g) genes. Data represent the average of data from two independent experiments, each conducted in triplicate. Error bars represent the mean \pm S.D. of six samples. ${ }^{*} P<0.05$ and ${ }^{* *} P<0.01$

According to data on the overexpression of CRBN, ubiquitination of TRAF6 and TAB2 were markedly attenuated, strongly indicating that CRBN-mediated inhibition of TLR4 signaling might be critically associated with the suppression of TRAF6 and TAB2 ubiquitination. We also confirmed the negative role of CRBN in TLR4 signaling by using CRBN ${ }^{\mathrm{KD}}$ THP-1 cells and CRBN-knockout mice. CRBN ${ }^{\mathrm{KD}}$ THP-1 cells showed enhanced NF- $k B$ activity and production of pro-inflammatory cytokines in response to TLR4 stimulation. As expected, the susceptibility to LPS challenge was significantly increased in the CRBN-knockout mice, accompanying elevated TNF and IL-6 levels, supporting the negative regulation of CRBN in TLR4 signaling.

There is a previously identified functional role of CRBN in the CRL4 ${ }^{\text {CRBN }}$ E3 ligase. ${ }^{6,13,14}$ Within the CRL4 ${ }^{\text {CRBN }}$ E3 ligase complex, DDB1 functions as the adaptor connecting the CRBN substrate receptor to the ligase. In regard to substrates for the CRL4 ${ }^{\mathrm{CRBN}}$ E3 ligase, endogenous substrates have been identified. ${ }^{6,13,14}$ For the ubiquitination of substrates by $\mathrm{CRL} 4^{\mathrm{CRBN}} \mathrm{E} 3$ ligase, the substrate that interacts with $\mathrm{CRBN}$ is recruited into the $C R L 4{ }^{\text {CRBN }}$ E3 ligase through the adaptor DDB1 protein, indicating that $\mathrm{CRBN}$ as a substrate receptor has a key role in the ubiquitination process. ${ }^{13}$ Importantly, a recent report has shown that $\mathrm{CRBN}$ functions via a ubiquitinindependent chaperone-like mechanism to mediate the folding and maturation of the CD147 and MCT1 proteins, thereby allowing activation of the CD147-MCT1 transmembrane complex. ${ }^{34}$ Interestingly, IMiDs abrogate this mechanism in a competitive manner to mediate their antitumor and teratogenic activities. The result has provided new insights into CRBN function and IMiD biology such as an ubiquitinindependent function for CRBN and as a new mode of action for IMiD. Moreover, CRBN-independent processes make a significant contribution to the anti-inflammatory properties of 
a

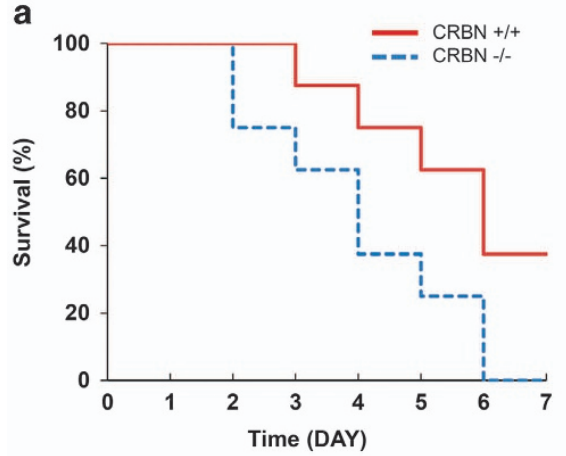

d

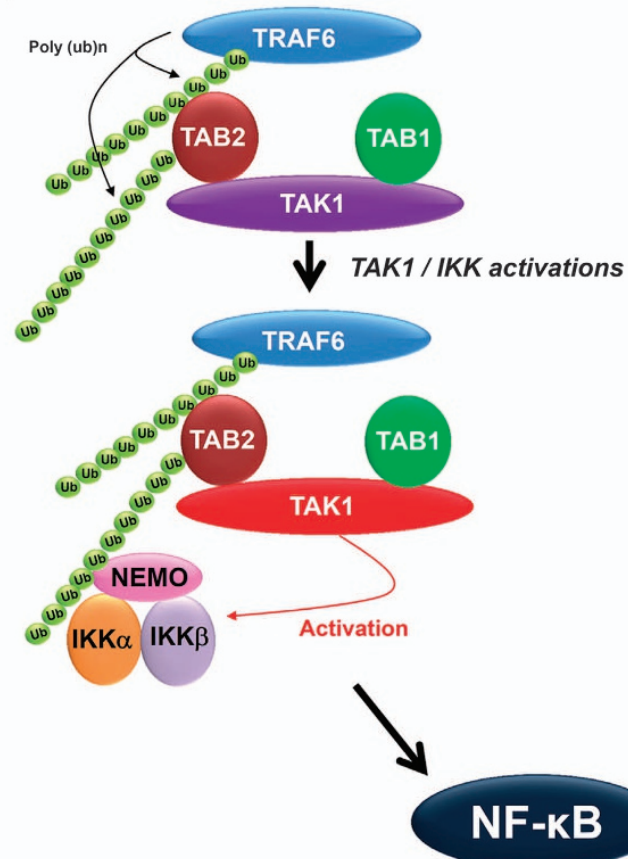

b

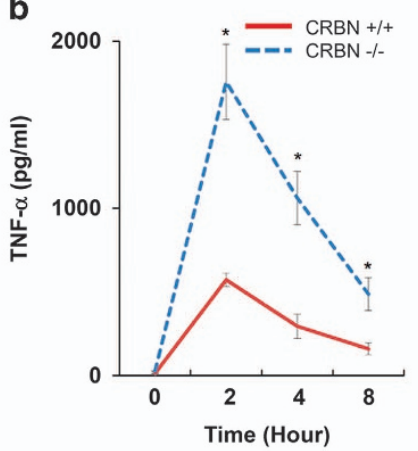

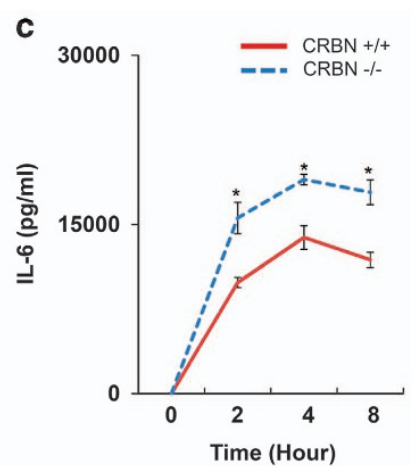

TRAF6
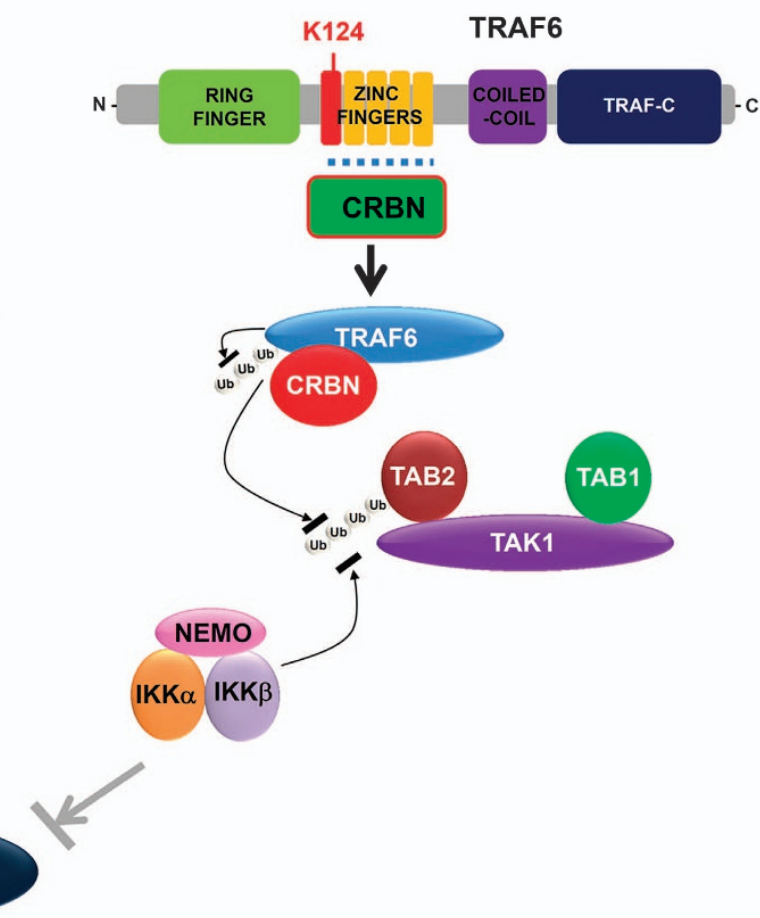

TRAF-C

Figure $7 \mathrm{Crbn}^{-1-}$ mice exhibit a higher mortality rate following LPS challenge. (a) $\mathrm{Crbn}^{-1-}(n=8)$ and $\mathrm{Crbn}^{+/+}(n=8)$ mice were injected intraperitoneally with $7.5 \mathrm{mg} / \mathrm{kg}^{\mathrm{k}}$ LPS in $100 \mu \mathrm{l}$ of PBS. Survival was monitored for 7 days after LPS challenge. (b,c) Serum from mice taken at different times after LPS challenge was isolated, and the concentrations of the TNF- $\alpha$ (b) and IL-6 (c) cytokines were measured with a BD Cytometric Bead Array. Error bars represent the mean \pm S.D. of eight samples. ${ }^{*} P<0.05$ (d) A schematic model of the negative regulation of CRBN in TLR4 signaling. Upon TLR4 stimulation, ubiquitinated TRAF6 is associated with the TAB2-TAK-TAB1 complex though the interaction between its poly-ubiquitinated chain and TAB2, TAB2 is ubiquitinated, and the complex facilities the activation of TAK1. Simultaneously, the IKK complex is associated with the former complex through the poly-ubiquitinated chain and TAB2, leading to the activation of IKKs (left). In contrast, the interaction between CRBN and TRAF6 may lead to the attenuation of ubiquitination of TRAF6 by masking the K124 residue of TRAF6 and inhibiting the association of the TAK1-TAB1-TAB2 complex to TRAF6, leading to inhibition of ubiquitination of TAB2 and decreased association of the IKK complex with ubiquitinated TAB2 (right)

thalidomide in mice. ${ }^{35}$ They showed that IMiDs are effective inhibitors of TLR4-induced type-1 interferon production via suppression of the TRIF/IRF3 pathway. Nevertheless, the molecular mechanisms by which CRBN or IMiDs mediate the anti-inflammatory effects have remained elusive. We demonstrate that CRBN masks the autoubiquitination site (K124) of TRAF6, and the CRBN-TRAF6 interaction inhibits TRAF6 ubiquitination, thereby resulting in inhibition of the recruitment of TAB2 to TRAF6 and the ubiquitination of TAB2. The inhibitory effect was critically associated with the suppression of NF- $k \mathrm{~B}$ activity, resulted in attenuations of the production of pro-inflammatory cytokines. Although it cannot be ruled out that IMiDs are implicated in the regulatory mechanism, we speculate that $C R B N$ itself functions as a binding protein to the autoubiquitination site of TRAF6, negatively regulating TLR4mediated signaling.

In terms of the regulation and control of immune responses, the negative regulators, like CRBN, may be very important. Since excessive or prolonged inflammatory responses to microbial infection can lead to harmful effects on the host, inflammatory signals including TLRs-related signaling need to be tightly controlled in the host. ${ }^{36}$ So far, various mechanisms and cellular proteins capable of interrupting TLRs-mediated signaling have been proposed and reported..$^{31,32}$ Negative regulation of TLRs signaling occurs in the membrane, cytoplasm, and nucleus through distinct inhibitors affecting 
multiple signaling steps. As mentioned above, the ubiquitination of TRAF6 critically affects downstream signaling molecules, indicating that TRAF6 ubiquitination is a plausible target for the control of TLRs-related signaling. Interestingly, various mechanisms by which TRAF6 is regulated have been discovered. ${ }^{37-41}$ A recent report showed that MST4 limits inflammatory responses through direct interaction and phosphorylation of the adaptor protein TRAF6. ${ }^{42}$ They found that modification of TRAF6 by MST4 induced impairments in homo-oligomeric association and thus the assembly of TRAF6-mediated signaling complexes, which in turn impeded K63-linked autoubiquitination of TRAF6 and downstream $N F-\kappa B$ signaling. These results strongly suggest that TRAF6 ubiquitination has a pivotal role in TLR-mediated signaling, thereby providing a potential cellular target for modulating excessive inflammatory responses under certain physiological conditions.

In conclusion, the ubiquitination of TRAF6 has an essential role for the association of the TAK1-TAB1-TAB2 complex via the interaction between the poly-ubiquitination chain of TRAF6 and TAB2 (Figure 7d, left). The association in turn leads to the ubiquitination of TAB2 and facilitates the activation of TAK1 and IKK complex. However, the interaction between CRBN and TRAF6 may lead to the attenuation of ubiquitination of TRAF 6 by masking the K124 residue of TRAF 6 and inhibit the association of the TAK1-TAB1-TAB2 complex to TRAF6, leading to inhibition of ubiquitination of TAB2 and the association of the IKK complex with ubiquitinated TAB2 (Figure 7d, right).

\section{Materials and Methods \\ Mice and animal experiments. CRBN-deficient mice $\left(\mathrm{Crbn}^{-1}\right)$ and control mice $\left(\mathrm{Crbn}^{+/+}\right)$were used as described previously. ${ }^{43}$ For LPS challenge, $\mathrm{Crbn}^{-1-}$ $(n=8)$ and $\mathrm{Crbn}^{+/+}(n=8)$ mice were injected intraperitoneally with $7.5 \mathrm{mg} / \mathrm{kg}$ LPS (Sigma-Aldrich, St. Louis, MO, USA) in $100 \mu$ l of phosphate buffered saline (PBS). Survival was monitored for 7 days after LPS challenge. To measure cytokines levels, sera was obtained from mice at different times after LPS challenge and the concentrations of the TNF- $\alpha$ and IL-6 cytokines were detected by BD Cytometric Bead Array (BD Biosciences, San Jose, CA, USA) according to the manufacturer's recommendations. All animal studies and protocols were approved by the Institutional Animal Use and Care Committee of Gwangju Institute of Science and Technology.}

Cells and antibodies. HEK293 cells expressing human TLR4 (293/TLR4) were purchased from InvivoGen (San Diego, CA, USA) and were maintained according to the manufacturer's protocol. HEK293T human embryonic kidney cells were purchased from the American Type Culture Collection (ATCC, Manassas, VA, USA) and were maintained in Dulbecco's modified Eagle's medium (DMEM; Invitrogen, Carlsbad, CA, USA) containing 10\% fetal bovine serum (FBS), $2 \mathrm{mM}$ L-glutamine, 100 units $/ \mathrm{ml}$ penicillin, $100 \mu \mathrm{g} / \mathrm{ml}$ streptomycin, and $5 \times 10^{-5} \mathrm{M}$ $\beta$-mercaptoethanol. $\mathrm{CRBN}^{+/+}$and $\mathrm{CRBN}^{-\gamma-}$ MEFs were isolated from E14.5 embryos born to heterozygous intercrosses, cultured in DMEM (Life Technologies, Carlsbad, CA, USA) with 10\% (v/v) FBS (Hyclone), and assayed at passages 3-6. THP-1 human monocytic cells were purchased from ATCC and were maintained in RPMI medium (Invitrogen) containing 10\% FBS, 2 mM L-glutamine, 100 units $/ \mathrm{m}$ penicillin, $100 \mu \mathrm{g} / \mathrm{ml}$ streptomycin, and $5 \times 10^{-5} \mathrm{M} \beta$-mercaptoethanol. Lentivirus containing small hairpin RNA (shRNA) targeting human CRBN (sc-78528-V), lentivirus containing shRNA targeting human TRAF6 (sc-36717-V), or control shRNA lentivirus (Sc-108080) were purchased from Santa Cruz Biotechnology (Santa Cruz, CA, USA). THP-1 cells were cultured in wells of a 24-well plate $\left(2 \times 10^{5}\right.$ cells per well) and infected with lentivirus according to the manufacturer's protocol. Ctrl THP-1 cells, CRBN-knockdown (CRBN ${ }^{\mathrm{KD}}$ ) THP-1 cells, and TRAF6knockdown (TRAFG ${ }^{\mathrm{KD}}$ ) THP-1 cells were selected in puromycin-containing $(4-8 \mu \mathrm{g} /$ $\mathrm{ml}$ ) medium and cultured as described previously. ${ }^{44,45}$ Specific anti-HA, anti-Flag,
anti-Myc, anti-TRAF6, and anti-GAPDH antibodies were from Cell Signaling Technology (Beverley, MA, USA). The anti-CRBN antibody was from Abcam (Cambridge, MA, USA). Mouse TrueBlot ULTRA: Anti-Mouse Ig HRP was from Rockland Immunochemicals Inc. (Limerick, PA, USA).

Luciferases reporter assay. 293/TLR4 cells, Ctrl THP-1, CRBN ${ }^{\mathrm{KD}}$ THP-1 cells, or TRAF ${ }^{K D}$ THP-1 cells were transiently transfected with different vectors, as indicated in the figures, using Lipofectamine LRX (Invitrogen) or the Neon transfection system (Invitrogen), together with the pBllx-luc NF- $\mathrm{KB}$-dependent reporter construct and the Renilla luciferase vector (Promega, Madison, WI, USA). At $24 \mathrm{~h}$ posttransfection, the cells were treated or not with LPS $(200 \mathrm{ng} / \mathrm{ml})$ for $6 \mathrm{~h}$ and lysed, and luciferase activity was measured using a dual luciferase assay kit (Promega).

Measurement of pro-inflammatory cytokines and p65- and p50DNA-binding assays. Wt THP- 1 , Ctrl THP-1, or CRBN ${ }^{\mathrm{KD}}$ THP-1 cells were untreated or treated with LPS $(200 \mathrm{ng} / \mathrm{ml})$ for $9 \mathrm{~h}$ and the supernatants were collected. The levels of human IL- $1 \beta$ and IL- 6 were measured in the supernatants according to the manufacturer's protocol (R\&D Systems, Minneapolis, MN, USA). Ctrl THP-1 and TRAF6 ${ }^{\mathrm{KD}}$ THP-1 cells were transfected with mock, Flag-TRAF6 wt, Flag-TAB2, and/or HA-CRBN, as indicated in each figure. Cell were untreated or treated with LPS $(200 \mathrm{ng} / \mathrm{ml})$ for $9 \mathrm{~h}$ and the supernatants were collected. The level of human IL-6 was measured in the supernatants according to the manufacturer's protocol (R\&D Systems). For p65- or p50-DNA-binding assay, cells were transiently transfected with mock or HA-CRBN vector. After $38 \mathrm{~h}$, nuclear proteins from transfectants treated for $6 \mathrm{~h}$ with or without LPS $(200 \mathrm{ng} / \mathrm{ml})$ were prepared with the Cellytic NuCLEAR extraction kit in accordance with the manufacturer's protocol (Sigma-Aldrich). Activities of the transcription factors p65 and $p 50$ were determined with the TransAM NF- $\kappa B$ transcription factor assay kit according to the manufacturer's instructions (Active Motif North America, Carlsbad, CA, USA).

Plasmids. HA-tagged CRBN, Flag-tagged CRBN, Myc-tagged CRBN, Myctagged TAK1, Flag-tagged TAK1, Flag-tagged TRAF6, Flag-tagged TAB1, Flagtagged TAB2, and HA-tagged Ub vectors were used. Myc-tagged TAK1 truncation mutants were generated as described previously. ${ }^{43}$ To generate Flag-tagged TRAF6 truncated mutants, specific primers were designed as described in Supplementary Table 1, and each truncated mutant was generated by PCR.

Western blotting and immunoprecipitation (IP) assay. Cells were transfected with the appropriate vectors, as indicated in each figure. Western blotting and IP assays were performed as described previously. ${ }^{46-49}$ The detailed procedures for the IP assay are described in Supplementary Materials and Methods. $\mathrm{CRBN}^{+/+}$or $\mathrm{CRBN}^{-/-}$MEF cells were stimulated without or with LPS for different times, lysed in lysis buffer, and the lysates were examined by western blotting with anti-pho-IKK $\alpha \beta$ (Cell Signaling Technology), anti-IKK $\alpha$ (Cell Signaling Technology), anti-IKB- $\alpha$ (Cell Signaling Technology), and anti- $\beta$-actin (Cell Signaling Technology) antibodies.

Ubiquitination assay. HEK293T cells were transiently transfected with MycCRBN, Flag-TRAF6, or Flag-TAB2, as indicated in the figures, along with an $\mathrm{HA}-\mathrm{Ub}$ vector. At $36 \mathrm{~h}$ after transfection, transfected cells were extracted and immunoprecipitated with an anti-Flag antibody. Immunoprecipitated complexes were separated by $6-10 \%$ SDS-PAGE and probed with anti-HA or anti-Flag antibody.

Microarray analysis. Microarray analysis, raw data preparation, and statistical analysis were performed as described in previous reports. ${ }^{45,48,50,51}$ Briefly, Ctrl and CRBN $^{K D}$ THP-1 cells were treated with or without LPS $(200 \mathrm{ng} / \mathrm{ml})$ for different times. Total RNA was isolated, and RNA purity and integrity were evaluated with an ND-1000 Spectrophotometer (NanoDrop Technologies, Wilmington, DE, USA) and an Agilent 2100 Bioanalyzer (Agilent Technologies, Palo Alto, CA, USA). Total RNA was amplified and purified using TargetAmp-Nano Labeling Kit for Illumina Expression BeadChip (EPICENTRE, Madison, WI, USA) to yield biotinylated cRNA according to the manufacturer's instructions. For the hybridization experiment, $750 \mathrm{ng}$ of labeled cRNA samples were hybridized to each Human HT-12 v4.0 Expression Beadchip for $17 \mathrm{~h}$ at $58{ }^{\circ} \mathrm{C}$, according to the manufacturer's instructions (Illumina, Inc., San Diego, CA, USA). Detection of array signal was carried out using fluorolink streptavidin-Cy3 (GE Healthcare Biosciences, Little Chalfont, UK) 
following the bead array manual. For raw data preparation and statistic analysis, the quality of hybridization and overall chip performance were monitored by visual inspection of both internal quality control checks and the raw scanned data. Raw data were extracted using the software provided by the manufacturer (Illumina GenomeStudio v2011.1 (Gene Expression Module v1.9.0); Illumina, San Diego, CA, USA)). Statistical significance of the expression data was determined using fold change. For a DEG set, hierarchical cluster analysis was performed using complete linkage and Euclidean distance as a measure of similarity. Gene-Enrichment and Functional Annotation analysis for a significant probe list was performed using DAVID (http://david.abcc.ncifcrf.gov/home.jsp). All data analysis and visualization of differentially expressed genes was conducted using R 3.0.2 (www.r-project.org).

Quantitative real-time polymerase chain reaction (qRT-PCR). Ctrl and CRBN ${ }^{\mathrm{KD}}$ THP-1 cells were treated with or without LPS $(200 \mathrm{ng} / \mathrm{ml})$ for different periods of time. Total RNA was isolated and cDNA was synthesized following the manufacturer's protocol (Qiagen, Erkrath, Germany). Primers (all from Qiagen) were: hlL-8 (PPH00568A-200), hIRF7 (PPH02014E-200), hCCL5 (PPH00703B-200), hBCL2 (PPH00079B-200), hIER3 (PPH10008F-200), and hlL-1 $\beta$ (PPH00171C-200). qRT-PCR analysis was performed using Rotor-Gene Q (Qiagen) according to the manufacturer's protocol.

Statistical analysis. In vitro data are presented as mean + S.D. of the mean from triplicate samples. Comparisons were statistically assessed using the Student's $t$-test. $P$ values $<0.05$ (or $<0.01$, as indicated) were considered to be statistically significant.

\section{Conflict of Interest}

The authors declare no conflict of interest.

Acknowledgements. We would like to thank the Hyehwa Forum members for their helpful discussions. This work was supported by the Mid-career Researcher Program through an NRF Grant (NRF-2014R1A2A1A11053221).

\section{Author contributions}

YM and SMW performed in vitro and ex vivo experiments. J-AK, TY, and S-GP performed and analyzed animal experiments. SC, S-GP, and J-HS analyzed and discussed data. EC and $\mathrm{K}-\mathrm{YL}$ designed the experiments and wrote the manuscript.

1. Higgins JJ, Pucilowska J, Lombardi RQ, Rooney JP. A mutation in a novel ATP-dependent Lon protease gene in a kindred with mild mental retardation. Neurology 2004; 63 . 1927-1931.

2. Basel-Vanagaite L, Attia R, Yahav M, Ferland RJ, Anteki L, Walsh CA et al. The CC2D1A, a member of a new gene family with $\mathrm{C} 2$ domains, is involved in autosomal recessive nonsyndromic mental retardation. J Med Genet 2006; 43: 203-210.

3. Jo S, Lee KH, Song S, Jung YK, Park CS. Identification and functional characterization of cereblon as a binding protein for large-conductance calcium-activated potassium channel in rat brain. J Neurochem 2005; 94: 1212-1224.

4. Hohberger $B, E n z R$. Cereblon is expressed in the retina and binds to voltage-gated chloride channels. FEBS Lett 2009; 583: 633-637.

5. Lee KM, Jo S, Kim H, Lee J, Park CS. Functional modulation of AMP-activated protein kinase by cereblon. Biochim Biophys Acta 2011; 1813: 448-455.

6. Ito T, Ando H, Suzuki T, Ogura T, Hotta K, Imamura Y et al. Identification of a primary target of thalidomide teratogenicity. Science 2010; 327: 1345-1350.

7. Sheskin J. Thalidomide in the treatment of lepra reactions. Clin Pharmacol Ther 1965; 6 : 303-306.

8. Singhal S, Mehta J, Desikan R, Ayers D, Roberson P, Eddlemon P et al. Antitumor activity of thalidomide in refractory multiple myeloma. N Engl J Med 1999; 341: 1565-1571.

9. Knobloch J, Rüther U. Shedding light on an old mystery: thalidomide suppresses survival pathways to induce limb defects. Cell Cycle 2008; 7: 1121-1127.

10. Miller MT, Strömland K. Teratogen update: thalidomide: a review, with a focus on ocular findings and new potential uses. Teratology 1999; 60: 306-321.

11. Parman T, Wiley MJ, Wells PG. Free radical-mediated oxidative DNA damage in the mechanism of thalidomide teratogenicity. Nat Med 1999; 5: 582-585.

12. D'Amato RJ, Loughnan MS, Flynn E, Folkman J. Thalidomide is an inhibitor of angiogenesis. Proc Natl Acad Sci U S A 1994; 91: 4082-4085.

13. Fischer ES, Böhm K, Lydeard JR, Yang H, Stadler MB, Cavadini S et al. Structure of the DDB1-CRBN E3 ubiquitin ligase in complex with thalidomide. Nature 2014; 512: 49-53.

14. Petzold G, Fischer ES, Thomä NH. Structural basis of lenalidomide-induced CK1 $\alpha$ degradation by the CRL4(CRBN) ubiquitin ligase. Nature 2016; 532: 127-130.
15. Mazzucotelli E, Belloni S, Marone D, De Leonardis A, Guerra D, Di Fonzo N et al. The e3 ubiquitin ligase gene family in plants: regulation by degradation. Curr Genomics 2006; 7 509-522.

16. Ardley HC, Robinson PA. E3 ubiquitin ligases. Essays Biochem 2005; 41: 15-30.

17. Zhang Y. Transcriptional regulation by histone ubiquitination and deubiquitination. Genes Dev 2003; 17: 2733-2740.

18. Liu YC. Ubiquitin ligases and the immune response. Annu Rev Immunol 2004; 22: 81-127.

19. Paolino M, Thien CB, Gruber T, Hinterleitner R, Baier G, Langdon WY et al. Essential role of E3 ubiquitin ligase activity in Cbl-b-regulated T cell functions. $J$ Immunol 2011; 186: 2138-2147.

20. Jin W, Chang M, Sun SC. Peli: a family of signal-responsive E3 ubiquitin ligases mediating TLR signaling and T-cell tolerance. Cell Mol Immunol 2012; 9: 113-122.

21. Janssens S, Beyaert R. Role of Toll-like receptors in pathogen recognition. Clin Microbiol Rev 2003; 16: 637-646.

22. West AP, Koblansky AA, Ghosh S. Recognition and signaling by toll-like receptors. Annu Rev Cell Dev Biol 2006; 22: 409-437.

23. Liu S, Chen ZJ. Expanding role of ubiquitination in NF-кB signaling. Cell Res 2011; 21 6-21.

24. Lamothe B, Besse A, Campos AD, Webster WK, Wu H, Darnay BG. Site-specific Lys-63linked tumor necrosis factor receptor-associated factor 6 auto-ubiquitination is a critical determinant of I kappa B kinase activation. J Biol Chem 2007; 282: 4102-4112.

25. Wang $\mathrm{C}$, Deng L, Hong M, Akkaraju GR, Inoue J, Chen ZJ. TAK1 is a ubiquitin-dependent kinase of MKK and IKK. Nature 2001; 412: 346-351.

26. Kim SY, Shim JH, Chun E, Lee KY. Reciprocal inhibition between the transforming growth factor-activated kinase 1 (TAK1) and apoptosis signal-regulating kinase 1 (ASK1) mitogenactivated protein kinase kinase kinases and its suppression by TAK1-binding protein 2 (TAB2), an adapter protein for TAK1. J Biol Chem 2012; 287: 3381-3391.

27. Kishida S, Sanjo H, Akira S, Matsumoto K, Ninomiya-Tsuji J. TAK1-binding protein 2 facilitates ubiquitination of TRAF6 and assembly of TRAF6 with IKK in the IL-1 signaling pathway. Genes Cells 2005; 10: 447-454

28. Moon G, Kim J, Min Y, Wi SM, Shim JH, Chun E et al. Phosphoinositide-dependent kinase-1 inhibits TRAF6 ubiquitination by interrupting the formation of TAK1-TAB2 complex in TLR4 signaling. Cell Signal 2015; 27: 2524-2533.

29. Wi SM, Moon G, Kim J, Kim ST, Shim JH, Chun E et al. TAK1-ECSIT-TRAF6 complex plays a key role in the TLR4 signal to activate NF-кB. J Biol Chem 2014; 289: 35205-35214.

30. Mi Wi S, Park J, Shim JH, Chun E, Lee KY. Ubiquitination of ECSIT is crucial for the activation of p65/p50 NF-kBs in Toll-like receptor 4 signaling. Mol Biol Cell 2015; 26: 151-160.

31. Shibolet O, Podolsky DK. TLRs in the Gut. IV. Negative regulation of Toll-like receptors and intestinal homeostasis: addition by subtraction. Am J Physiol Gastrointest Liver Physiol 2007 292: G1469-G1473.

32. Liew FY, Xu D, Brint EK, O'Neill LA. Negative regulation of toll-like receptor-mediated immune responses. Nat Rev Immunol 2005; 5: 446-458.

33. Ajibade AA, Wang HY, Wang RF. Cell type-specific function of TAK1 in innate immune signaling. Trends Immunol 2013; 34: 307-316.

34. Eichner R, Heider M, Fernández-Sáiz V, van Bebber F, Garz AK, Lemeer S et al. Immunomodulatory drugs disrupt the cereblon-CD147-MCT1 axis to exert antitumor activity and teratogenicity. Nat Med 2016; 22: 735-743.

35. Millrine D, Miyata H, Tei M, Dubey P, Nyati K, Nakahama T et al. Immunomodulatory drugs inhibit TLR4-induced type-1 interferon production independently of Cereblon via suppression of the TRIF/IRF3 pathway. Int Immunol 2016; 6: 307-315.

36. Beutler B. Inferences, questions and possibilities in Toll-like receptor signalling. Nature 2004; 430: 257-263.

37. Trompouki E, Hatzivassiliou E, Tsichritzis T, Farmer H, Ashworth A, Mosialos G. CYLD is a deubiquitinating enzyme that negatively regulates NF-kappaB activation by TNFR family members. Nature 2003; 424: 793-796.

38. Boone DL, Turer EE, Lee EG, Ahmad RC, Wheeler MT, Tsui C et al. The ubiquitin-modifying enzyme A20 is required for termination of Toll-like receptor responses. Nat Immunol 2004 5: $1052-1060$

39. Kobayashi K, Hernandez LD, Galán JE, Janeway CA Jr, Medzhitov R, Flavell RA. IRAK-M is a negative regulator of Toll-like receptor signaling. Cell 2002; 110: 191-202.

40. Yuk JM, Shin DM, Lee HM, Kim JJ, Kim SW, Jin HS et al. The orphan nuclear receptor SHP acts as a negative regulator in inflammatory signaling triggered by Toll-like receptors. Nat Immunol 2011; 12: 742-751.

41. Zhang X, Zhang J, Zhang L, van Dam H, ten Dijke P. UBE2O negatively regulates TRAF6mediated NF-KB activation by inhibiting TRAF6 polyubiquitination. Cell Res 2013; 23 366-377.

42. Jiao S, Zhang Z, Li C, Huang M, Shi Z, Wang Y et al. The kinase MST4 limits inflammatory responses through direct phosphorylation of the adaptor TRAF6. Nat Immunol 2015; 16 246-257.

43. Lee KM, Yang SJ, Kim YD, Choi YD, Nam JH, Choi CS et al. Disruption of the cereblon gene enhances hepatic AMPK activity and prevents high-fat diet-induced obesity and insulin resistance in mice. Diabetes 2013; 62: 1855-1864

44. Kim SY, Chun E, Lee KY. Phospholipase A(2) of peroxiredoxin 6 has a critical role in tumor necrosis factor-induced apoptosis. Cell Death Differ 2011; 18: 1573-1583.

45. Wi SM, Min Y, Lee KY. Charged MVB protein 5 is involved in T-cell receptor signaling. Exp Mol Med 2016; 29: e206. 
46. Kim SY, Jo HY, Kim MH, Cha YY, Choi SW, Shim JH et al. $\mathrm{H}_{2} \mathrm{O}_{2}$-dependent hyperoxidation of peroxiredoxin 6 ( $\mathrm{Prdx} 6$ ) plays a role in cellular toxicity via up-regulation of PRLA2 activity. J Biol Chem 2008; 283: 33563-33568.

47. Kim SY, Kim TJ, Lee KY. A novel function of peroxiredoxin 1 (Prx-1) in apoptosis signalregulating kinase 1 (ASK1)-mediated signaling pathway. FEBS Lett 2008; 582: 1913-1918.

48. Kim SY, Baik KH, Baek KH, Chah KH, Kim KA, Moon G et al. S6K1 negatively regulates TAK1 activity in the toll-like receptor signaling pathway. Mol Cell Biol 2014; 34: 510-521.

49. Wi SM, Lee KY. 5-Aminoimidazole-4-carboxamide riboside induces apoptosis through AMP-activated protein kinase-independent and NADPH oxidase-dependent pathways. Immune Netw 2014; 14: 241-248.

50. Oh SJ, Ahn JY, Chung DH. Comparison of invariant NKT cells with conventional T cells by using gene set enrichment analysis (GSEA). Immune Netw 2011; 11: 406-411.

51. Kim SY, Jeong S, Jung E, Baik KH, Chang MH, Kim SA et al. AMP-activated protein kinase- $\alpha 1$ as an activating kinase of TGF- $\beta$-activated kinase 1 has a key role in inflammatory signals. Cell Death Dis 2012; 26: e357. (c) (i) Cell Death and Disease is an open-access journal published by Nature Publishing Group. This work is licensed under a Creative Commons Attribution 4.0 International License. The images or other third party material in this article are included in the article's Creative Commons license, unless indicated otherwise in the credit line; if the material is not included under the Creative Commons license, users will need to obtain permission from the license holder to reproduce the material. To view a copy of this license, visit http://creativecommons.org/licenses/by/4.0/

(C) The Author(s) 2016

Supplementary Information accompanies this paper on Cell Death and Disease website (http://www.nature.com/cddis) 